\title{
Study on the Natural Frequency Characteristics of Lift Gate Vibration Based on Added Mass Model
}

\author{
Shihua $\mathrm{He}^{\mathrm{a}}$, Jianjun Zhu ${ }^{\mathrm{b}}$, Chunying $\mathrm{Shen}^{\mathrm{c}}$ and Tingting Yang ${ }^{\mathrm{d}}$ \\ Faculty of Electric Power Engineering, Kunming University of Science and Technology, Kunming, \\ 650500, China \\ ahoxiwa@aliyun.com, b360273491@qq.com, '86295700@qq.com, d912832062@qq.com
}

Keywords: Plane lift gate; Added mass; Dynamic characteristics; Natural frequency

Abstract: The dynamic characteristics of plane lift gate are analyzed based on the model of added mass arising from flow field which is induced by gate vibration in static water. The effect of added mass caused by fluid-solid coupling on gate natural frequency is considered. The natural frequency characteristics of plane gate with or without water and at different opening, different water head is investigated. The numerical simulating results show that effect of added water mass on the gate dynamic characteristics is greatly and leads to significantly lower natural frequency than the gate without water. The larger the gate opening is, the higher the natural frequency of gate vibration is. When the upstream or downstream head of gate is increased, the natural frequency will decrease.

\section{Introduction}

Vibration problems of plane lift gate often arise during its opening and closing process or in partial, and even in fully closed state. When the vibration reaches a certain extent, it will cause damage to the gate and the surrounding hydraulic buildings. Although external reasons of gate vibration are different, but the internal causes lie in its natural vibration characteristics

For the gate in the fluid-structure interaction system, the effect of water should be considered in analyzing gate dynamic characteristics. Using finite element method, Literature [1] established the discrete models of fluid and structure domains respectively, introduced coupling conditions in the corresponding nodes located the fluid-solid interface, and implemented accurately transfer of water pressure load on the fluid-solid interface. The simulating method based on fluid-structure theory has some problems such as too large amount of calculations, numerical implementation difficulties and difficult to determine the scope of the fluid domain. When the structure amplitude is smaller, the impact of structural vibration on fluid domain is also smaller and the fluid-structure coupling effect can be simplified into considering the added mass of coupling system. it has more engineering significance to introduce the added mass model to reflect the impact of water on self-excited vibration of structure [2].

The added mass model established by literature [3] is applied to analyze the dynamic characteristics of a plane lift gate in present work. The change trends of gate natural frequency obtained under different working conditions can provide a reference for design and safe operation of the lift gate.

\section{Numerical models}

For a fluid-structure interaction system, when the structure amplitude is smaller, the impact of structural vibration on fluid domain is also smaller and the fluid-structure coupling effect can be simplified into using the added mass, added damping and added stiffness to describe. Considering fluid effects, the control equation of structural vibration can be written as[4, 5]

$$
\left[M_{s}+M_{a}\right]\left\{\&+\left[C_{s}+C_{a}\right]\{\mathcal{Q}\}+\left[K_{s}+K_{a}\right]\{U\}=\{0\}\right.
$$

Where $\left[M_{s}\right],\left[C_{s}\right]$ and $\left[K_{s}\right]$ are structure mass, damping and stiffness matrices respectively. $\left[M_{a}\right],\left[C_{a}\right]$ and $\left[K_{a}\right]$ are added mass, damping and stiffness matrices generated by fluid acting on the structure. $\{\& \&\},\{U\}\}$ and $\{U\}$ are node acceleration, velocity and displacement vector respectively. 
If the influence of fluid field on structure stiffness ignored, then $\left[K_{a}\right]=0$. At the same time, the less influence of ideal fluid on damping force can be ignored. The discrete structure dynamic control equation (1) is simplified as

$$
\left[M_{s}+M_{a}\right]\left\{\left\{+\left[K_{s}\right]\{U\}=\{0\}\right.\right.
$$

According to potential flow theory and singularity distribution method[6], Literature [3] deduced the expression (3) of added mass $M_{a}$ arising from fluid movement induced by gate vibration.

$$
M_{a}^{(r)}=-\rho \sum_{j=1}^{m} \sum_{j=1}^{m} \mu_{i}^{(r)} \iint_{A_{j}} \varphi_{i}^{(r)} \psi_{i}^{(r)} \stackrel{V}{n} d A
$$

Where $i, j=1,2, \mathrm{~L}, m, m$ the number of element divided on the fluid-structure interface. $\vec{n}$ is the normal direction of element $j, \rho$ the fluid density. $\psi_{i}^{(r)}$ is the orthonormalized principal modal vector corresponding $r$-order natural frequency of the structure. $\mu_{i}^{(r)}$ is a scaling factor. $\varphi_{i}^{(r)}$ is the basic solutions for space point sources ( sinks ) and dipole of unit intensity

The main steps of gate dynamic characteristics analysis based on the added mass model are as follows.

(1) Determine the added mass.

(2) Extract the element nodes on fluid-structure coupling interface.

(3) Determine added mass size of $r$-order vibration mode for each node and define the mass element.

(4) Calculate the $r$-order natural dynamic characteristics of gate and extract the results. If the required number of modes met, the calculation is terminated. Otherwise, redefine the added mass of $(r+1)$-order vibration mode, turn to (3).

Literature [3] analyzed the validity and accuracy based on the added mass expression (3) to calculate the dynamic characteristics of plane gate.

The dynamic characteristics for a gate of plate size $4.4 \mathrm{~m} \times 4.6 \mathrm{~m} \times 14 \mathrm{~mm}$ (width $\times$ height $\times$ thickness) are analyzed in present work. The gate material is Q235. $E=2.0 \times 10^{5} \mathrm{MPa}$ is elasticity modulus. $\mu=0.3$ is Poisson ratio. $\rho=7850 \mathrm{~kg} / \mathrm{m}^{3}$ is mass density. $\rho=1000 \mathrm{~kg} / \mathrm{m}^{3}$ is Water density. $v=1460 \mathrm{~m} / \mathrm{s}$ is velocity of sound in water. The plane gate structure is divided into 4144 elements and 4076 nodes. Finite element model of gate structure is shown in fig. 1 . The $x$-, $y$ - and $z$-axes are respectively set as along gate span, gate height and the water flow directions.

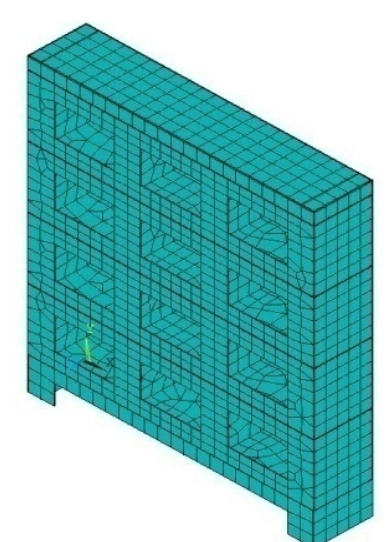

Fig.1 Finite element model of lift gate

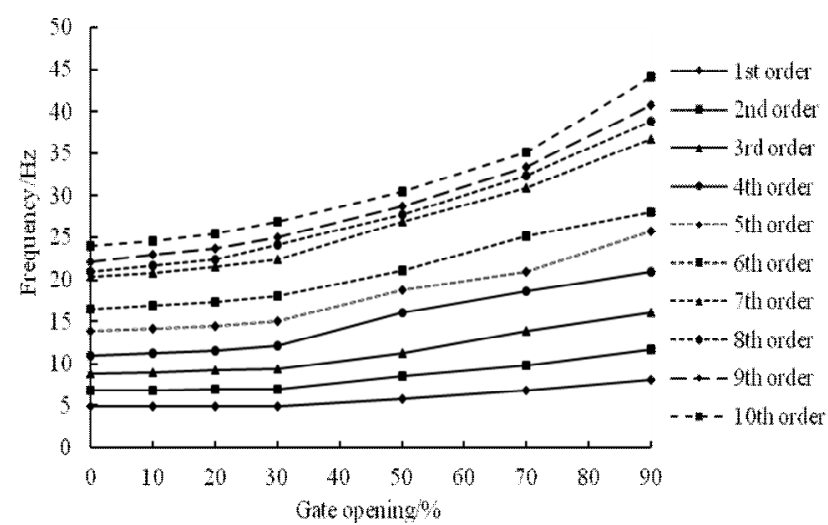

Fig. 2 Relation between frequency and opening

\section{Results and discussion}

According to the actual operating conditions of gate, the upstream and downstream heads of gate are set as $80 \mathrm{~m}$ and $30 \mathrm{~m}$ respectively. Firstly, the dynamic characteristics of four working conditions 
WC1, WC2, WC3, and WC4 of the gate in the fully closed state are analyzed. WC1, WC2, WC3, and WC4 represent the gate without water and without lateral restraint, with water and without lateral restraint, without water and with lateral restraint, and with water and with lateral restraint respectively.

Table 1 The first ten order natural frequencies $[\mathrm{Hz}]$ of gate under different conditions

\begin{tabular}{ccccccccccc}
\hline order & 1 & 2 & 3 & 4 & 5 & 6 & 7 & 8 & 9 & 10 \\
\hline WC1 & 13.602 & 18.396 & 18.971 & 22.739 & 27.914 & 30.689 & 39.649 & 40.680 & 42.843 & 44.670 \\
WC2 & 4.876 & 6.743 & 8.871 & 11.026 & 13.845 & 16.427 & 20.332 & 21.008 & 22.154 & 23.982 \\
WC3 & 15.732 & 17.624 & 18.772 & 22.845 & 28.157 & 30.285 & 39.627 & 40.549 & 43.011 & 44.685 \\
WC4 & 4.891 & 6.786 & 8.942 & 11.109 & 13.958 & 16.517 & 20.387 & 21.159 & 22.276 & 24.219 \\
\hline
\end{tabular}

The first ten order natural frequencies of the gate under different working conditions are shown in table 1. It can be seen from the data in table 1 that water is of great influence on the natural frequency of the gate and leads to significantly lower natural frequencies than those in the air. No matter whether to consider the lateral restraint of gate. Among the 1st to 10th order natural frequencies, the reduction of the first order frequency is the largest. For the conditions $\mathrm{WC} 1$ and $\mathrm{WC} 2$ of gate without lateral restraint, the first order natural frequency of gate in water is only $35.85 \%$ of that in the air. For the conditions WC3 and WC4 of gate subjected to lateral restraint, the first order natural frequency of gate in water is only $31.09 \%$ of that in the air. Average value of the first ten order natural frequencies of the gate in the air is about twice those in water. These illustrate the fluid-structure interact significantly affects the vibration characteristics of the gate, and the added water mass can't be ignored

Comparing the results of the conditions WC1 and WC3 of gate without water, it can be seen that the lateral restraint has only a small effect on the natural frequency. Although the relative difference of the first order natural frequency is up to $15.66 \%$, but all relative difference from the second to tenth orders is only about $1 \%$. Comparing the results of the conditions WC2 and WC4 of gate with water, it can be seen that the lateral restraint has a smaller effect on the natural frequency and the relative difference of each order natural frequency is less than $1 \%$. The main reason of the effect of lateral restraint on vibration frequency so small lies in that the gate vibration is mainly along the water flow direction, namely the $z$-direction, while lateral restraint is exerted along the $x$-direction.

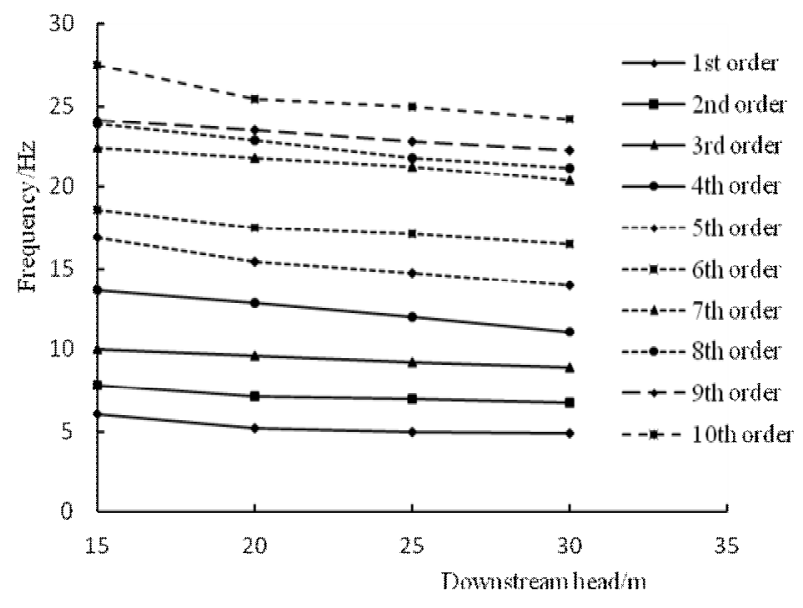

Fig. 3 Relation between frequency and downstream head

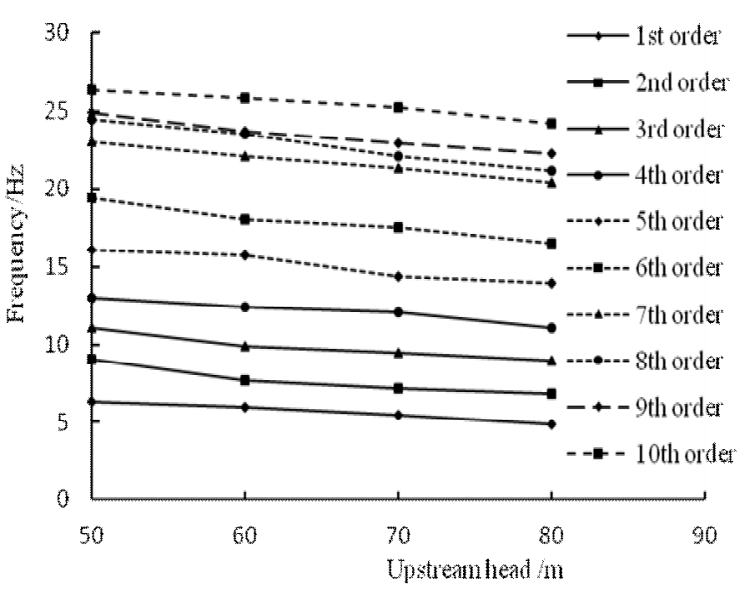

Fig. 4 Relation between frequency and upstream head

When the gate in water is partially opened, Fig. 2 shows the results of the first ten order natural frequencies under different gate opening. It can be seen that the general trend of each order natural frequency of the gate is increased with the gate opening increasing. The main reason is that the larger the gate opening, the smaller the gate-water coupling area and added water mass effect, the higher the natural frequency of gate vibration.

When the upstream head is $80 \mathrm{~m}$ and the downstream heads are respectively set as $15 \mathrm{~m}, 20 \mathrm{~m}, 25 \mathrm{~m}$, and $30 \mathrm{~m}$, the first ten order natural frequencies of the gate in the fully closed state are shown in Fig. 3. 
When downstream head is $30 \mathrm{~m}$ and the upstream heads are respectively set as $50 \mathrm{~m}, 60 \mathrm{~m}, 70 \mathrm{~m}$, and $80 \mathrm{~m}$, the first ten order natural frequencies of the gate in the fully closed state are shown in Fig. 4. It can be seen that both the upstream and downstream heads increase, the natural frequencies of the gate will be decreased. The fundamental cause is also that the higher the water head, the greater the added water mass effect, the lower the natural frequency of gate vibration.

\section{Conclusions}

Unlike the wing-induced vibration of structure, added mass is an important physical variable for water-structure coupling vibration problem and significantly affects the vibration characteristics of the structure. The natural frequency of the gate vibration in water is significantly lower than that in the air, wherein the reduction of the first order natural frequency is the largest. Because of the gate vibration mainly along the water flow direction, the effect of lateral restraint on the natural frequencies is small. With the decreasing of the gate opening and the increasing of water head, the natural frequency of gate vibration is decreased, which shows that the larger water-gate coupling area is, the greater added water mass effect is, and the lower the natural frequency of gate vibration is.

\section{Acknowledgements}

The financial supports from the National Natural Science Foundation (51369013) and the Natural Science Foundation of Kunming University of Science and Technology (KKZ3201304016) are gratefully acknowledged.

\section{References}

[1] M. Xu, H.F. Xue, Research on self-vibration characteristics of plane gate based on ANSYS [J], Machine Building \& Automation, 41(1) (2012):109-110, 155.

[2] L. Cao, L.X. Zhang, Additive mass method for dynamical property of the fixed parts of Francis turbine [J], China Rural Water and Hydropower, (2) (2008): 107-109.

[3] J.J. Zhu, S.H. He, L.T. Wu, M. Fan, The equation of added mass from fluid field induced by flat gate in still water [J], China Rural Water and Hydropower, (12) (2014): 118-120.

[4] C.C. Liang, The free vibration analysis of submerged cantilever plates [J], Ocean Eng., 28(9) (2001): 1225-1245.

[5] M. Amabili, G. Frosali, M.K. Kwak, Free vibration of annular plates coupled with fluid [J], J. Sound Vibrat., 191(5) (1996): 825-846.

[6] X.F. Sun, S.L. Dong, Numerical analysis of added mass arising from fluid flow induced by vibration of three-dimensional structure [J], Engineering Mechanics, 25(7) (2008): 1-4. 